\title{
Structure and spectra of irradiated secondaries in close binaries
}

\section{A model calculation of the pre-cataclysmic variable UU Sagittae}

\author{
A. C. Wawrzyn ${ }^{1,2}$, T. S. Barman ${ }^{2}$, H. M. Günther ${ }^{1}$, P. H. Hauschildt ${ }^{1}$, and K. M. Exter ${ }^{3}$ \\ 1 Hamburger Sternwarte, Gojenbergsweg 112, 21029 Hamburg, Germany \\ e-mail: awawrzyn@hs .uni-hamburg.de \\ 2 Lowell Observatory, Hendricks Center for Planetary Studies, 1400 W. Mars Hill Rd., Flagstaff, AZ 86001, USA \\ e-mail: barman@lowell.edu \\ 3 Instituut voor Sterrenkunde, Celestijnenlaan 200 D, 3001 Heverlee (Leuven), Belgium
}

Received 4 February 2009 / Accepted 24 June 2009

\section{ABSTRACT}

\begin{abstract}
Context. The standard stellar model atmosphere ignores the influence of external radiation. This assumption, while sufficient for most stars, fails for many short-period binaries.

Aims. In setting up combined model atmospheres for close binaries, we want to constrain the parameters of both components, especially in the case of a hot primary component strongly influencing its cool secondary companion. This situation can be found after common envelope evolution (CEE). The status of both components today allows one to retrace the CEE itself.

Methods. We used our stellar atmosphere code PHOENIX, which includes the effect of irradiation in its radiation transport equation, to investigate the close binary star UU Sge. We combined our calculated spectra of both components, weighted by their visible size, and adjusted the input parameters until reasonable agreement with observations is reached.

Results. We derive a range of $80000-85000 \mathrm{~K}$ for the effective temperature of the primary $\left(T_{\text {eff, }}\right)$ and give a rough estimate for the primary's abundances, particularly the nitrogen enrichment. The heated day-side of the secondary has an apparent "effective" or equilibrium temperature of $24000-26000 \mathrm{~K}$, nearly independent of its intrinsic luminosity. It shows an enhancement in nitrogen and carbon.

Conclusions. The evolution of the primary and secondary stars were strongly influenced by the other's presence. Radiation from the primary on the secondary's day-side is still an important factor in understanding the secondary's atmospheric structure.
\end{abstract}

Key words. radiative transfer - stars: abundances - binaries: eclipsing - binaries: close - stars: atmospheres stars: individual: UU Sge

\section{Introduction}

UU Sagittae (UU Sge) is the central nucleus of the old planetary nebula (PN) Abell 63 (Abell 1966). This nucleus is a total-eclipsing close binary that has passed through the commonenvelope phase and is currently a pre-cataclysmic variable (pre$\mathrm{CV}$ ). The primary has been classified as an $\mathrm{O}$ subdwarf ( $\mathrm{sdO}$ ) that has not yet contracted to a white dwarf (WD) (Bond et al. 1978). The secondary companion is thought to be an unevolved main-sequence star (MS) by its mass, probably a mid K- to mid M-dwarf (dKV-dMV), but the luminosity of the night-side, due to horizontal heat transfer beyond the terminator, is comparable to that of a late A- to early F-star (dAV-dFV). The PN is faint but still detectable, where a typical PN lifetime before dispersion is about $3 \times 10^{4}$ years (de Kool \& Ritter 1993; Iben \& Tutukov 1993). The PN spectrum is unusual in that the H Balmer series, O III (5007, 4959, and $4363 \AA$ ) and He I $5876 \AA$ are the only strong lines (Miller et al. 1976). It should hence not contaminate the observed spectra of the nucleus. UU Sge's unique "totally eclipsing" nature allows one to determine well-constrained lightcurve solutions and, in combination with accurate radial velocity data, the derivation of reliable geometrical parameters for the system. Even though the geometry is well constrained, different quality measurements and diverse physical implications have produced a variety of mass and temperature estimates in the past, as discussed in Sect. 2.

The reasonably well constrained geometry and observationally accessible nature of UU Sge makes this system a useful laboratory for studying the effects of irradiation in a close binary. The system's hot primary, the cooler secondary and the proximity of both components make the effects of irradiation an important feature. A crucial aspect of UU Sge is that none of the indications associated with mass transfer (e.g. accretion disk, bright spot, or boundary layer) are observed, which would otherwise make the irradiation geometry asymmetric and far more difficult to characterise.

Another important feature of UU Sge is that the primary sdO is much larger than a fully evolved WD; the size of the primary is almost comparable to that of the companion. Therefore the estimated $T_{\text {eff }}$ ratio of $\sim 90000 \mathrm{~K}: 6000 \mathrm{~K}$ by Pollacco \& Bell (1993, hereafter PB93) and Bell et al. (1994, hereafter BPH94) leads directly to a luminosity ratio of approximately $10^{4}$ between the primary and the (faint) night-side of the secondary close to the primary eclipse.

The geometry allows the fundamental parameters of the primary, i.e., $T_{\text {eff, }}, \log g_{\mathrm{p}}$ and chemical composition, to be decoupled from the secondary, even though they are not spatially resolved in the observation. The primary near its eclipse can be dealt with in a first step, neglecting the very small influence of 
the secondary for the moment. The primary spectrum can then be used to irradiate the secondary to model its day-side in a second step, since reflection and heating effects from the secondary on the primary should be negligible.

The sum of primary and secondary spectrum must reproduce the emission near the secondary eclipse, where its heated dayside contributes significantly to the total flux; this defines the properties of the secondary.

In the following we do not concentrate on the common envelope evolution (CEE) (e.g. Paczynski 1976; Iben \& Livio 1993; Warner 1995; Livio 1996; Taam \& Sandquist 2000). This is the mechanism that is thought to expel the envelope of the Asymptotic Giant Branch (AGB) star (Rasio \& Livio 1996; Sandquist et al. 1998) and due to this momentum loss produce close binaries; though the CEE surely has influenced what we observe in UU Sge today.

UU Sge has conserved its properties basically unaltered since the end of the CEE, because it is a pre-CV and no other major physical processes such as additional mass accretion has taken place yet. However, there are ongoing discussions upon this topic and alternative momentum loss mechanisms are suggested by e.g. Nelemans \& Tout (2005); Taam \& Ricker (2006); Webbink (2007); Beer et al. (2007).

For a review about detached binaries, physical processes in close binary systems, and general three-dimensional fluid dynamics in binary systems see Marsh (2000); Claret \& Giménez (2001); Beer \& Podsiadlowski (2002).

The paper is structured as follows: In Sect. 2 we summarise the properties of UU Sge and in Sect. 3 we present the observation that we model. Section 4 contains a short description of the stellar atmosphere code PHOENIX and the assumptions made for starting values. Section 5 presents the results for primary and secondary, followed by a discussion in Sect. 6. Section 7 concludes with a summary.

\section{Properties of UU Sge}

The first to suggest that UU Sge was an eclipsing binary was Hoffleit (1932), who found it only twice at minimum on 25 plates. More than 30 years later UU Sge was listed as an eclipsing binary in the PN catalogue of Abell (1966); however, not until Bond (1976) found the PN Abell 63 and the variable star UU Sge at the same position was the true nature of the system established. Early predictions for the system parameters were published by Budding \& Kopal (1980), Budding (1981), and Ritter (1986). Others followed shortly after. Further improvements were made by PB93 who measured the effective temperature $\left(T_{\text {eff, }}\right)$ of the primary to be $87000 \mathrm{~K}$ and improved radial velocities that indicated an oversized secondary ( 2.0-2.5 times larger than a corresponding zero-age MS star) leading to important changes to the inferred geometry.

One year later the secondary was, for the first time, observed directly during a total primary eclipse, which lasts some $14 \mathrm{~min}$. The time between first and last contact with the primary is $1.26 \mathrm{~h}$. This measurement provided an intrinsic temperature of $6250 \mathrm{~K}$ for the secondary's unilluminated night-side (BPH94). There is an ongoing effort to constrain system parameters further (e.g. more recent work by Pustynski \& Pustylnik 2005; Afşar \& Ibanoğlu 2008) since the uncertainties still do not allow the evolutionary status to be pinpointed.

Table 1 shows a compilation of previously derived parameters of UU Sge from Bond et al. (1978), de Kool \& Ritter (1993), Walton et al. (1993), PB93/BPH94, Pustynski \& Pustylnik (2005), Afşar \& Ibanoğlu (2008), and our results for comparison.
We used Kepler's 3rd law to fill in missing separations where possible. We use, like Pustynski \& Pustylnik (2005), the geometry derived by PB93. Numbers in round brackets in the last column are fixed input parameters and not derived by our model. Values in squared brackets are only used outside the model calculation. Note that we do not list a value of $T_{\text {eff, } p}=120000 \mathrm{~K}$ by Shimansky et al. (2008), which is discussed at a later point.

The parameters are (top to bottom) visual magnitude $m_{V}$ [mag], distance to Earth $d$ [pc], luminosity $L\left[L_{\odot}\right]$, mass $m\left[M_{\odot}\right]$, radius $r\left[R_{\odot}\right]$, logarithmic surface gravity $\log g\left[\mathrm{~cm} \mathrm{~s}^{-2}\right]$, effective temperature $T_{\text {eff }}[\mathrm{K}]$ for primary ( $)$ and secondary (s), equilibrium temperature $T_{\text {eq }}[\mathrm{K}]$ on day-side of secondary, separation $R$ (centre to centre) $\left[R_{\odot}\right]$, mass ratio $q$ of components, period $P[\mathrm{~h}]$, and system inclination $i\left[^{\circ}\right]$, where $90^{\circ}$ is edge on. Note that many of these parameters are strongly coupled and, even though most ratios are well determined, an error in, e.g., radial velocity does not only affect the separation but also the radii and masses of the system and, consequently, other parameters such as gravity, luminosity, and irradiation.

The effective temperatures of both components are especially difficult to determine and a case can be made for higher as well as lower temperatures, using e.g. the excitation of the PN or missing opacities of other lines when using Balmer line ratios (Exter et al. 2005, and references therein). If cooler temperature estimates are correct, the primary may be a non-degenerate helium remnant of a star of initial mass of about $5 M_{\odot}$ (Iben \& Tutukov 1989). If instead hotter temperature estimates turn out to be true, the hot component of UU Sge could be a star with a degenerate $\mathrm{CO}$ core and a non-degenerate helium envelope which is burning helium at its base (Iben \& Tutukov 1993). According to Iben \& Tutukov (1993) a luminosity of $19000 L_{\odot}$ found by PB93 is likely too high, since it exceeds the Eddington limit for a star of that predicted mass; they suggest $10^{3}-10^{4} L_{\odot}$.

\section{Observations}

Figure 1 shows two spectra (continuum normalised) of UU Sge obtained during phases close to primary and secondary eclipse along with the difference in flux. The wavelength coverage is $4185-4770 \AA$.

Data were taken with the spectrograph ISIS of the William Herschel Telescope and first published in PB93, where details on the observations and data reduction procedures are given. Radial velocity shifts close to the eclipses are unimportant since the movement is perpendicular to line-of-sight. Stellar velocity shifts were corrected to the theoretical wavelengths.

We define phase $\varphi=0.0$ (inferior conjunction) to coincide with primary eclipse, where the larger secondary occults the primary and orients its non-irradiated side to the observer. At phase $\varphi=0.5$ (superior conjunction) a secondary eclipse occurs as the smaller primary transits the heated day-side of the secondary. The observations were taken close to but not exactly at $\varphi=0.0$ and $\varphi=0.5$, so both components were visible during the exposures (PB93). The first spectrum shows the primary and the cold night-side of the secondary. The second spectrum also includes the primary but this time with the hot day-side of the secondary. The bottom panel in Fig. 1 displays the difference between hot day-side and cold night-side of the secondary, since the primary should roughly cancel out.

Identified H I, He II, and N V absorption features are marked, where "IS" means the unknown diffuse interstellar band at $4430 \AA$ that was not taken into account for the fitting of our model spectra. 
Table 1. A selection of former derived values of UU Sge.

\begin{tabular}{|c|c|c|c|c|c|c|c|c|}
\hline Parameter & Units & Bond & Ritter & Walton & Pollacco & Pustynski & Afşar & This paper \\
\hline$m_{\mathrm{v}}$ & [mag] & 15 & n.a. & n.a. & 14.67 & n.a. & n.a. & n.a. \\
\hline$d$ & {$[\mathrm{pc}]$} & 150-2000 & n.a. & $\sim 3600$ & $2400 \pm 400$ & n.a. & n.a. & [2400] \\
\hline$L$ & {$\left[L_{\odot}\right]$} & 100 & n.a. & $\sim 2400$ & $(19000)^{*}$ & n.a. & n.a. & [3000-6000] \\
\hline$m_{\mathrm{p}}$ & {$\left[M_{\odot}\right]$} & 0.9 & 0.7 & 0.565 & $0.63 \pm 0.06$ & $0.63 \pm 0.06$ & $0.628 \pm 0.053$ & $(0.63)$ \\
\hline$r_{\mathrm{p}}$ & {$\left[R_{\odot}\right]$} & 0.4 & n.a. & n.a. & $0.33 \pm 0.01$ & $0.33-0.34$ & $0.349 \pm 0.013$ & $(0.34)$ \\
\hline $\log g_{\mathrm{p}}$ & {$\left[\mathrm{cm} \mathrm{s}^{-2}\right]$} & n.a. & n.a. & 5.0 & $5.18 \pm 0.05$ & n.a. & $5.151 \pm 0.050$ & $4.5-5.0$ \\
\hline$T_{\mathrm{eff}, \mathrm{p}}$ & {$[\mathrm{K}]$} & $14000-60000$ & n.a. & $\sim 50000$ & $87000 \pm 13000$ & $80000-85000$ & $78000 \pm 3000$ & $75000-85000$ \\
\hline$m_{\mathrm{s}}$ & {$\left[M_{\odot}\right]$} & 0.7 & 0.5 & 0.7 & $0.29 \pm 0.04$ & $0.29 \pm 0.04$ & $0.288 \pm 0.031$ & $(0.29)$ \\
\hline$r_{\mathrm{s}}$ & {$\left[R_{\odot}\right]$} & 0.7 & 0.6 & n.a. & $0.53 \pm 0.02$ & 0.54 & $0.558 \pm 0.021$ & $(0.53)$ \\
\hline $\log g_{\mathrm{s}}$ & {$\left[\mathrm{cm} \mathrm{s}^{-2}\right]$} & n.a. & n.a. & n.a. & $4.43 \pm 0.06$ & n.a. & $4.404 \pm 0.058$ & $(4.5)$ \\
\hline$T_{\mathrm{eff}, \mathrm{s}}$ & {$[\mathrm{K}]$} & n.a. & n.a. & n.a. & $6250 \pm 250$ & $5500-5600$ & $6136 \pm 240$ & [6000] \\
\hline$T_{\text {eq, } \mathrm{s}}$ & {$[\mathrm{K}]$} & $\sim 10000$ & n.a. & n.a. & $17000 \pm 2000$ & n.a. & $31000 \pm 1000$ & $23000-26000$ \\
\hline$R$ & {$\left[R_{\odot}\right]$} & $\sim 3$ & 2.98 & n.a. & {$[2.46]$} & n.a. & $2.45 \pm 0.09$ & $(2.46)$ \\
\hline$q=m_{\mathrm{s}} / m_{\mathrm{p}}$ & & n.a. & n.a. & n.a. & 0.46 & n.a. & $0.46 \pm 0.034$ & n.a. \\
\hline$P$ & {$[\mathrm{~h}]$} & 11.16 & 11.161656 & n.a. & 11.162 & n.a. & 11.162 & [11.162] \\
\hline$i$ & {$\left[{ }^{\circ}\right]$} & n.a. & n.a. & n.a. & n.a. & $88^{\circ}$ & $87.12 \pm 0.19$ & {$\left[87.5^{\circ}\right]$} \\
\hline
\end{tabular}

Bond et al. (1978); de Kool \& Ritter (1993); Walton et al. (1993); PB93/BPH94; Pustynski \& Pustylnik (2005); Afşar \& Ibanoğlu (2008) and this paper.

* See comment of Iben \& Tutukov (1993) at the end of Sect. 2.

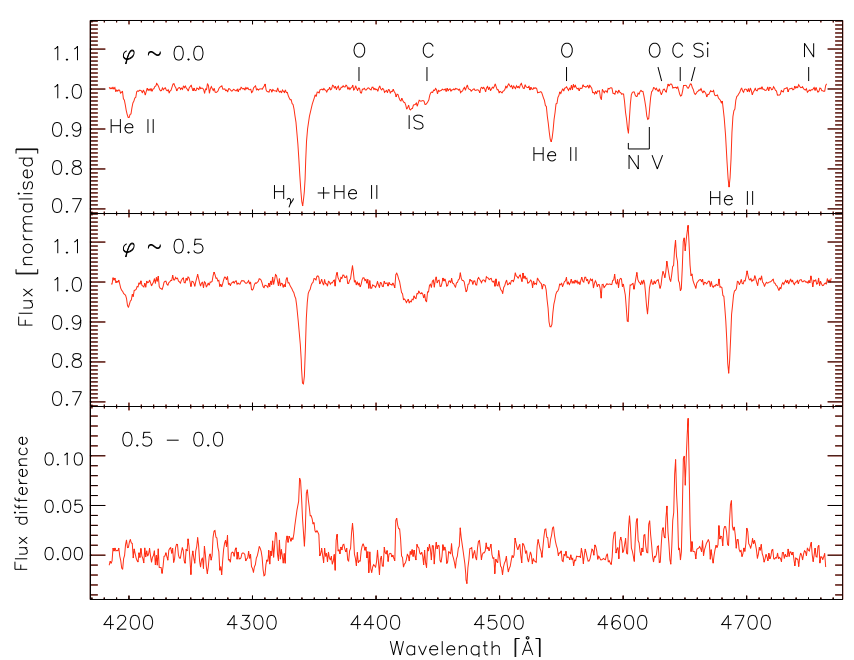

Fig. 1. Observed spectrum (PB93) close to the primary eclipse (top), close to the secondary eclipse (middle), and the difference spectrum (bottom).

Also indicated are where our synthetic spectra predict lines. In particular, we indicate the lines O IV $\lambda 4390.7,4555.8$,

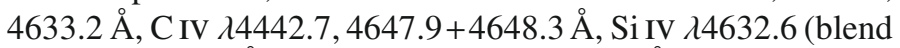
with O), $4655.6 \AA$ and N v $\lambda 4750.1+4751.8 \AA$.

As is evident in the bottom panel of Fig. 1, only two wavelength ranges are especially interesting with strong "differential" emission remaining from the secondary's day-side: a double feature around $4340 \AA$ A, which results from emission "filling-in" in the $\mathrm{H}_{\gamma}$ wings and a multiple feature between 4630 and $4655 \AA$. These are investigated in Sect. 5.2 in more detail.

\section{The model}

\subsection{The code}

We use a modified version of the PHOENIX 15.04 stellar atmosphere code (Hauschildt \& Baron 1999) for the calculations presented here. This is capable of modelling both the hot sdO (Aufdenberg 2001) and the cooler MS companion (Allard et al. 2001). The code includes an irradiation mode
(Barman et al. 2004, 2005), which allows the outer boundary conditions of the radiation transport equation to include the incoming intensities from a primary star. The primary spectrum is a full stellar spectrum, not a simple black body. The most important lines, namely $\mathrm{H}, \mathrm{He}, \mathrm{C}, \mathrm{N}$, and $\mathrm{O}$, are treated with full non-local thermodynamic equilibrium (NLTE) during the calculations. Other species, e.g. Fe and $\mathrm{Mg}$, were reset to LTE to save computation time once we tested their influence on the model. The model considers the distance between both stellar components, their radii, and the angle between incident flux and surface normal. The parameters were fixed to the set of PB93/BPH94. We use our spherical symmetric radiation transport (SSRT) mode for the secondary, whose atmosphere is divided into 64 concentric shells and $\tau_{\text {std }}$ is set to $5000 \AA$.

To obtain the spectrum of the secondary two simulations are independently carried out: the irradiated day-side; and the nightside, which resembles a MS star. The day-side is assumed to re-radiate all the incident flux, so we use a geometric scaling factor of 0.5 . The lateral energy transport between the day- and night-side of the secondary is assumed to be negligible. The irradiation heats and expands the photosphere of the day-side, so we assume in our model that thermodynamic variables like entropy and gas pressure are discontinuous at the boundary between dayside and night-side for a given stellar radius. Consequently, small scale turbulence will develop in a boundary layer, which is not included in our model.

\subsection{Setup}

For all currently derived radii and distances between the components the secondary still underfills its Roche lobe by about $30 \%$ (see e.g. Eggleton 1983; Pringle \& Wade 1985), i.e., there is no mass transfer to the primary. The secondary should hence be reasonably well approximated by a sphere, which justifies the use of our SSRT mode.

The primary most likely lost a large fraction of its $\mathrm{H}$ and He envelope during its CEE, while the secondary accreted additional material during that state. Since hotter post-early-AGB stars tend to be sdOs with a normal $\mathrm{H} / \mathrm{He}$ ratio (Moehler et al. 1998) we use the solar standard composition for the initial condition. 
The metallicity $Z$ of both components is based on the solar photospheric abundances by Asplund et al. (2005). Further variations will be dealt with during the model fit (Sects. 5.1 and 5.2).

The temperature of sdOs ranges from $T_{\text {eff, }}=40000$ to $100000 \mathrm{~K}$ and the surface gravity from $\log g_{\mathrm{p}}=4.5$ to 6.5 (Dreizler \& Murdin 2000). Since UU Sge seems to be a rather young sdO it should tend to higher $T_{\text {eff, }}$ and $\operatorname{lower} \log g_{\mathrm{p}}$, i.e., it has neither cooled nor contracted much yet. For the companion it is reasonable to assume an ordinary MS star with $T_{\mathrm{eff}, \mathrm{s}}=3400 \mathrm{~K}$ and $\log g_{\mathrm{s}}=4.5$, where the last value results from the inferred mass and radius of the secondary by BPH94. However, it is heated to higher temperatures on the day-side, where a horizontal heat flux towards the night-side probably also effectively doubles the temperature (BPH94 measure temperatures of the order $6000 \mathrm{~K}$ on the night-side). We take the above values as an initial guess.

Fundamental parameters of the primary were explored by calculating a grid of effective temperatures $\left(T_{\text {eff, }}\right)$, surface gravities $\left(\log g_{\mathrm{p}}\right)$, and metallicities $\left(Z_{\mathrm{p}}=\log [\mathrm{H} / \mathrm{M}]\right)$. The fitting was then done by comparing five selected strong features to the observation close to the primary eclipse. We identified residuals and adapted the parameters accordingly, to improve the fit iteratively.

The secondary is assumed to be an ordinary MS star and is then irradiated by primary spectra of different temperatures. The influence of varying $T_{\text {eff }}$ of both components and abundances is investigated.

Finally, we note that we do not include a wind or chromosphere in the model calculations.

\section{Results}

\subsection{The primary}

We fit the primary model to the observation at $\varphi \approx 0$. 0 , i.e., close to primary eclipse, where the flux of the secondary's night-side is negligible and virtually all flux originates from the primary.

The lines are rotationally broadened. Velocities were first calculated assuming a tidally locked and circular orbit, with $2 \pi r_{\mathrm{p}} / P=36.9 \mathrm{~km} \mathrm{~s}^{-1}$ for the primary, which, however, requires a $v \sin i=160 \pm 20 \mathrm{~km} \mathrm{~s}^{-1}$ depending on the major feature probed. This value is high but still well below the breakup velocity of $v=\sqrt{\frac{G M}{r}} \approx 600 \mathrm{~km} \mathrm{~s}^{-1}$. Note that a velocity field, due to the mass loss caused by a strong wind, might cause macro turbulence in the line forming regions that mimics rotational broadening to some extend.

For the primary this comparison shows that the $\mathrm{N} \mathrm{V}$ absorption features agree well with the observations for $\log g_{\mathrm{p}}=$ 5.0-5.5 and $T_{\text {eff,p }}=70000-75000 \mathrm{~K}$. This is right between the limb darkening solution $(57000 \mathrm{~K})$ and the limb brightening solution $(87000 \mathrm{~K})$ of BPH94. Grid points with higher $T_{\text {eff, }}$, closer to the light curve analysis of Pustynski \& Pustylnik (2005) and compatible with $\mathrm{PB} 93$, require an increase of the $\mathrm{N}$ abundance $\epsilon_{\mathrm{N}}$ by +1.5 dex and more. $\mathrm{N}$ is normally increased by less than one order of magnitude in AGB evolution (van Winckel 2003). Also emission of C IV from the secondary's day-side suggests that the primary is not much hotter than $85000 \mathrm{~K}$ or further away from the secondary than the assumed $2.46 R_{\odot}$ separation (see Sect. 5.2). Note, that our model of the secondary's day-side represents the spectrum of the substellar point only and the constraint on the primary temperature could be less severe if the secondary's day-side is a "patchwork" of different temperature.
Table 2. Primary abundances with respect to solar values*.

\begin{tabular}{lcccccc}
\hline \hline & $\mathrm{H}$ & $\mathrm{He}$ & $\mathrm{C}$ & $\mathrm{N}$ & $\mathrm{O}$ & $\mathrm{Si}_{(\mathrm{LTE})}$ \\
\hline Figure 2 & 12.00 & 10.53 & 8.19 & 8.98 & 8.56 & 7.81 \\
$75000 \mathrm{~K}$ & $($ fix $)$ & -0.4 & -0.2 & +1.2 & -0.1 & $\leq+0.3$ \\
\hline Figure 3 & 12.00 & 10.73 & 8.19 & 9.28 & 8.56 & 7.81 \\
$85000 \mathrm{~K}$ & $($ fix $)$ & -0.2 & -0.2 & +1.5 & -0.1 & $\leq+0.3$ \\
\hline Figure 4 & 12.00 & 10.43 & 8.19 & 9.38 & 8.56 & 7.81 \\
$85000 \mathrm{~K}^{* *}$ & $($ fix $)$ & -0.5 & -0.2 & +1.6 & -0.1 & $\leq+0.3$ \\
\hline
\end{tabular}

* Based on Asplund et al. (2005).

** Reduced $\log g_{\mathrm{p}}=4.5$ to match He II $\lambda 4686$.
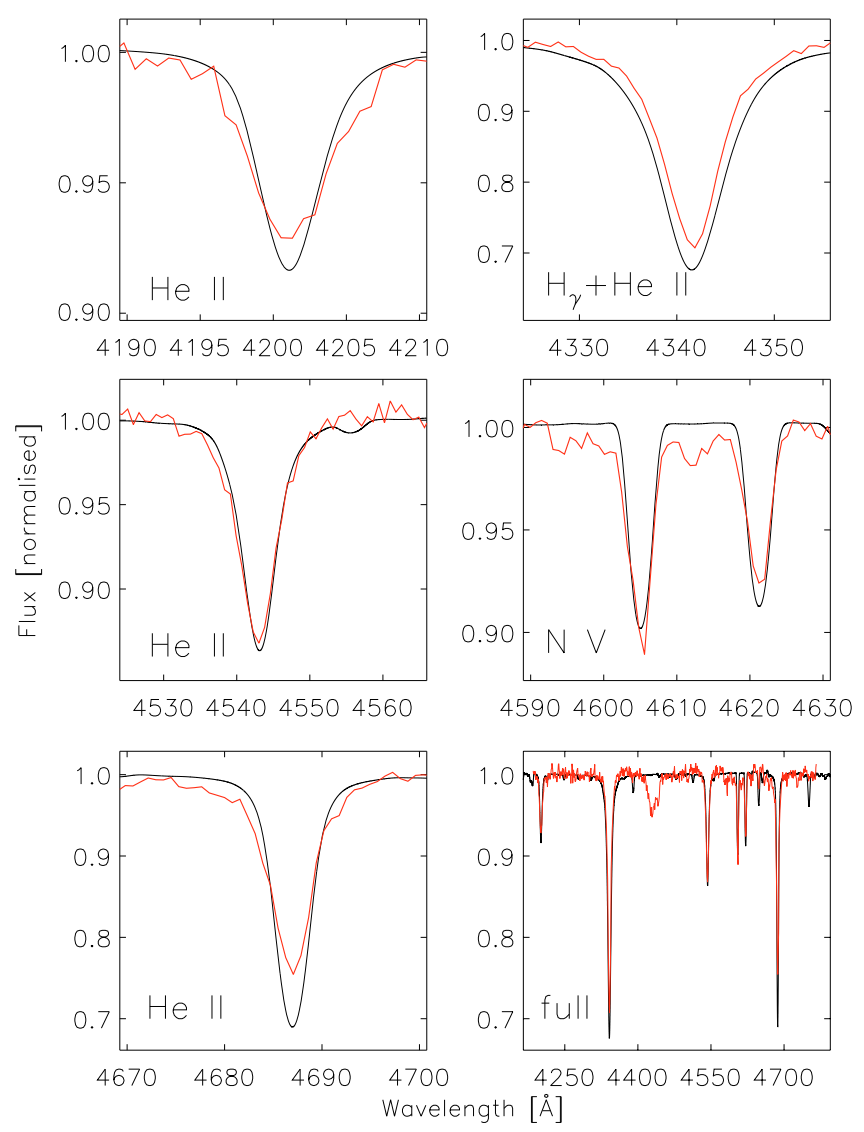

Fig. 2. A synthetic spectrum for the primary with $T_{\text {eff, } p}=75000$, $\log g_{\mathrm{p}}=5.0$ and abundances from Table 2 . The first five figures show particular absorption features and the sixth displays the full observed range for an overview. Displayed are observation at $\varphi \sim 0.0$ (red/grey) and synthetic spectrum (black).

The intensities of the N V $\lambda 4603,4619 \AA$ absorption lines in comparison to the C IV $\lambda 4441 \AA$ and the O IV $\lambda 4631 \AA$ lines suggest that $\mathrm{N}$ is overabundant and $\mathrm{C}$ and $\mathrm{O}$ are underabundant in the atmosphere of the primary. Si is only a minor species and not handled in NLTE. Our best-fit abundances for a model with $T_{\text {eff, }}=75000 \mathrm{~K}$ are listed in Table 2 .

The general abundance pattern is best described by $Z_{\mathrm{p}}=$ +0.5 , however, our model only derives the ratios of these elemental abundances, so selecting a specific $Z_{\mathrm{p}}$ is somewhat arbitrary. For all our models the $\mathrm{N}$ abundance is enormous, while $\mathrm{C}$ and $\mathrm{O}$ seem to be slightly depleted. Si only requires depletion for a base metallicity of +0.5 dex.

Figure 2 shows a comparison between the best fit primary synthetic spectrum with $T_{\text {eff, } p}=75000, \log g_{\mathrm{p}}=5.0$ and abundances from Table 2, and the observation close to phase $\varphi=0.0$. The night-side spectrum of the secondary was not calculated at 

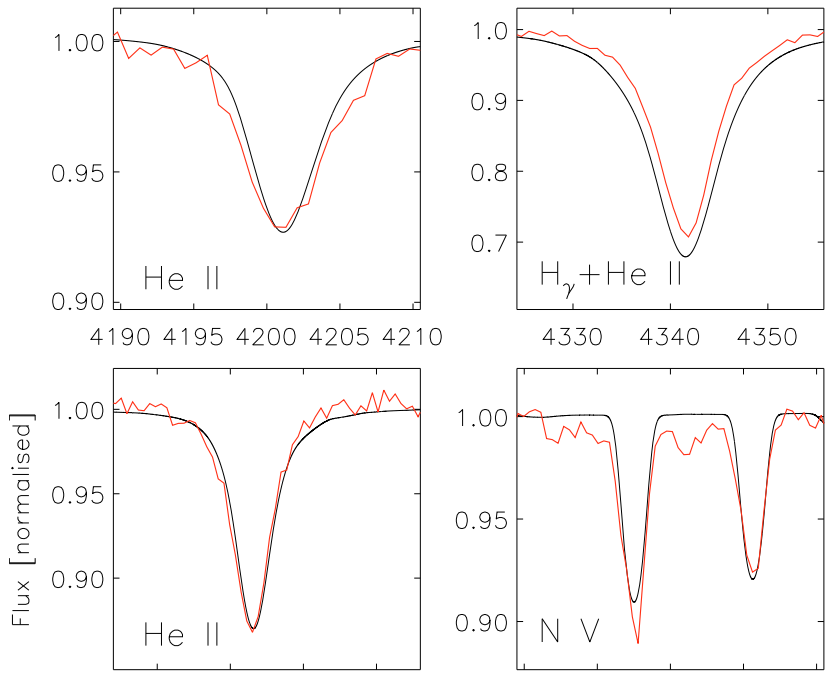

4530454045504560

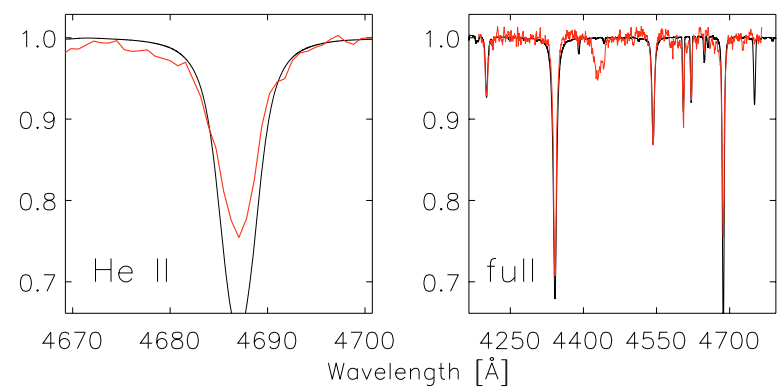

Fig. 3. As Fig. 2, but for $T_{\text {eff, } \mathrm{p}}=85000, \log g_{\mathrm{p}}=5.4$ and abundance deviations from Table 2 .

this point and hence not added to the synthetic spectrum of the primary. Displayed are solely the features of the primary.

Figure 3 also shows a comparison between observation and synthetic spectrum, but for a model with $T_{\text {eff, }}=85000$, $\log g_{\mathrm{p}}=5.4$ and the abundances of Table 2. The quality of the fit is similar to the previous model but requires a higher $\epsilon_{\mathrm{N}}$ and produces stronger unobserved $\mathrm{N}$ lines.

The He II absorption feature at $4686 \AA$ is systematically too strong in our synthetic spectra. This feature is characteristic for most sdOs. In our model it depends only weakly on the He abundance or temperature. Contrary to the other He lines it increases in depth for higher $\log g$ values. It is not blended with other lines.

Using the mass and radius from PB93 $\log g_{\mathrm{p}}$ of the primary is constrained to 5.2 . However, the $\lambda 4686 \mathrm{He}$ line fits the observation best for $\log g_{\mathrm{p}}=4.5$, as can be seen in Fig. 4. This fit also requires a He depletion of -0.5 dex to match the other two He lines. All important He II absorption features of the primary within the observed spectral range are dealt with using special Stark line profiles (Hubeny \& Lanz 2000), so the effect of $\log g$ should be real, suggesting a much lower $\log g_{\mathrm{p}}$ than given by PB93. Note, that adding the day-side spectrum of the secondary contributes some emission that reduces the problem, while the night-side spectrum influence can be neglected as expected.

In Fig. 5 the variation of the absorption line depth with $T_{\text {eff, }}$ is shown. In order to match the observed line depth, the $\mathrm{N}$ abundance had to be increased greatly with increasing $T_{\text {eff, }}$. This allows us to constrain the temperature range to values of $70000-85000 \mathrm{~K}$ : the N V (3s-3p) doublet starts to go into emission for higher $T_{\text {eff, }}$. The other two features not displayed in Fig. $5, \mathrm{H}_{\gamma}+\mathrm{He}$ II $\lambda 4338$ and He II $\lambda 4686$, depend only weakly on
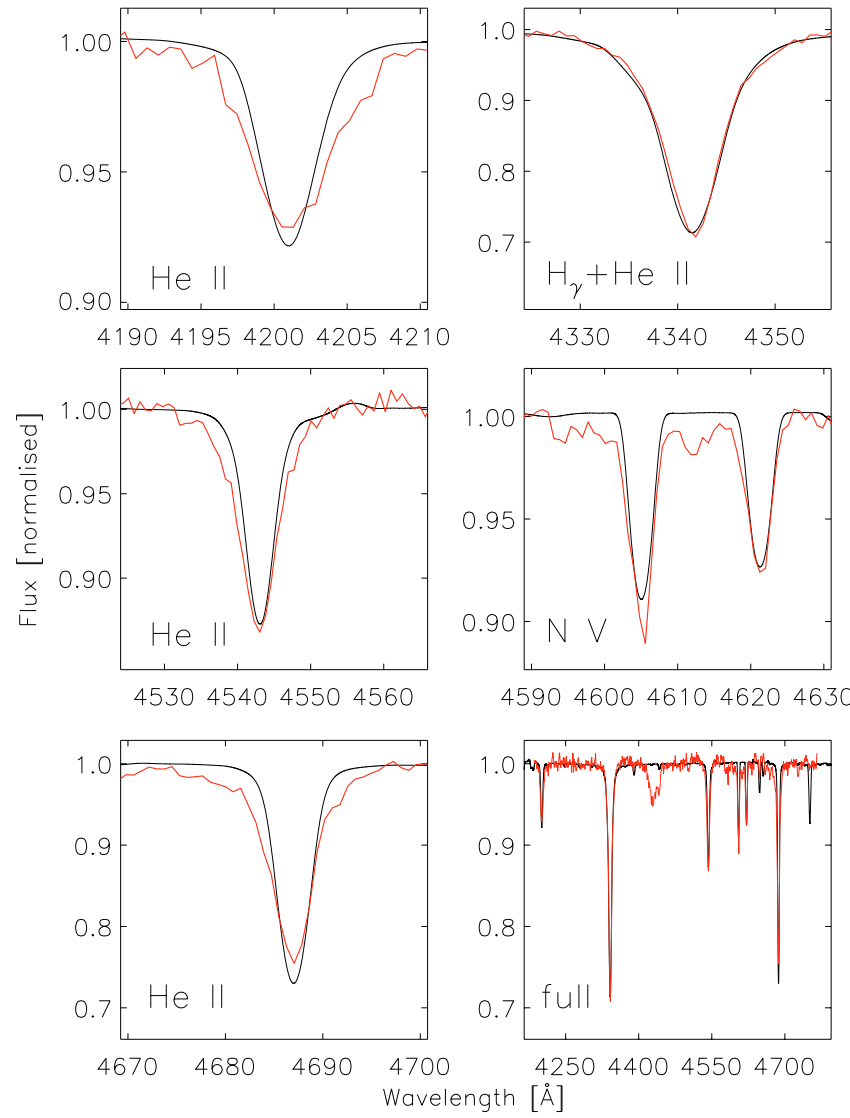

Fig. 4. As Fig. 2, but for $T_{\text {eff, } \mathrm{p}}=85000, \log g_{\mathrm{p}}=4.5$ and abundance deviations from Table 2 .
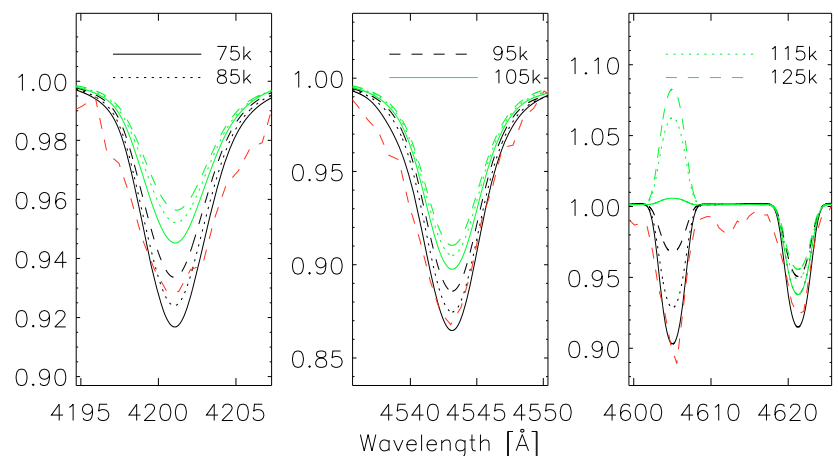

Fig. 5. Variation in lines due to increase of $T_{\text {eff, }}$. All other parameters are fixed to best-fit values of Fig. 2. The observation is also shown (red/gray).

temperature. The $\lambda 4338$ absorption decreases slightly with increasing temperature. All He lines, except for $\lambda 4686$, are weakened by increasing $\log g$.

If the primary parameter ranges are fixed, we can get a rough approximation for the equilibrium temperature due to irradiation $T_{\text {eq, s }}$ on the secondary's day-side by simple physical considerations (Exter et al. 2005), i.e., what temperature of a nonirradiated model reproduces the increased luminosity:

$T_{\mathrm{eq}, \mathrm{s}}=T_{\mathrm{eff}, \mathrm{p}} \sqrt{\frac{r_{\mathrm{p}}}{\left(R-r_{\mathrm{s}}\right)}} \approx 30000 \mathrm{~K}$

where we used $T_{\text {eff, }}=75000 \mathrm{~K}$ and the derived geometry of Table 1. This is valid if the irradiation influence (extrinsic luminosity) dominates the energy flux from the core (intrinsic 

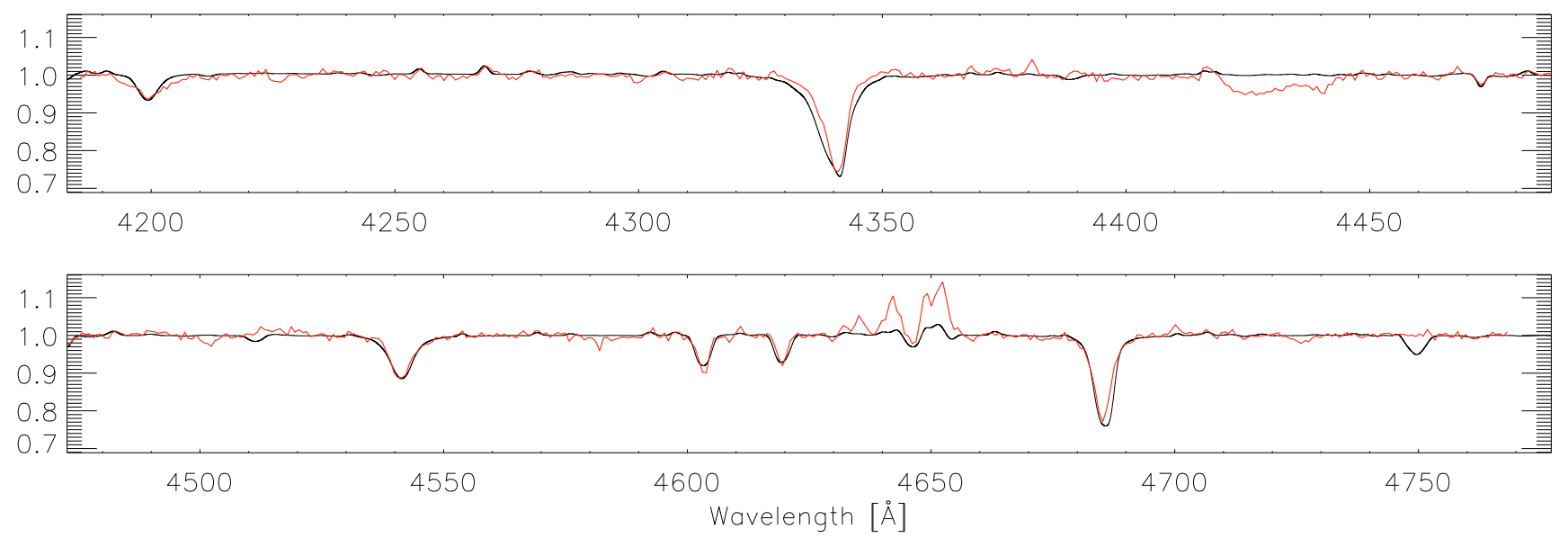

Fig. 6. Combined theoretical spectrum of the best-fit primary and the initial secondary day-side model, using the primary model ( $85000 \mathrm{~K}$, increased abundance from Table 2 by another $\epsilon_{\mathrm{N}}=+0.4$ dex and $\epsilon_{\mathrm{He}}=+0.1$ dex) to irradiate a $T_{\mathrm{eff}, \mathrm{s}}=3400 \mathrm{~K}, \log g_{\mathrm{s}}=4.5, Z_{\mathrm{s}}=0$ secondary, where $\mathrm{C}, \mathrm{N}$, and $\mathrm{O}$ are handled in LTE only. The synthetic spectrum (black) vs. observation (red/gray).

luminosity) and the energy is reprocessed, i.e. absorbed and re-emitted. In reality some of the incoming irradiation will be reflected directly and not reprocessed, so lower values for $T_{\text {eq, s }}$ are possible.

\subsection{The secondary}

We fit the day-side of the secondary component to the difference spectrum of phase $\varphi \approx 0.5$ minus $\varphi \approx 0.0$, i.e., subtract the primary's influence on the total spectrum. Since there are not many emission lines left, we test the overall influence and then focus on the "broad emission feature" at 4635-4655 $\AA$. This feature is uniquely sensitive to the abundances used.

All absorption lines are Doppler-shifted in anti-phase with the emission features, which shows that the first originate from the primary while the others are due to irradiation on the hot side of the secondary. This is confirmed by the phase dependence of the emission lines, which are strongest at phases close to 0.5 and not visible at all near phase 0.0 .

In Fig. 6 the combined best-fit primary and initial secondary spectrum is shown. The observed extra emission "filling-in" the wings of $\mathrm{H} \gamma$ is too weak (top panel) to explain the differences seen in the bottom panel of Fig. 1. There are other small emission lines in the synthetic spectrum which cannot be found in the observation. In the bottom panel of Fig. 6 the broad emission feature at $4635-4655 \AA$ is not reproduced well by solar abundance in the secondary model, since important C III and N III lines are too weak (see Fig. 7 for details). Also emissions lines such as N II $\lambda 4602.8, \lambda 4603.0, \lambda 4603.8$, O II $\lambda 4603.3$, and C II $\lambda 4619.9$, $\lambda 4620.5$ originating from the secondary start to balance the primary $\mathrm{N} v$ absorptions significantly more than observed.

These all indicate that the heating effect on the secondary is higher than first estimated and that the abundances of $\mathrm{C}, \mathrm{N}$, and probably $\mathrm{O}$ need to be increased. We used $Z=+0.5 \mathrm{dex}$ and varied $T_{\text {eff, }}$ to match the broad emission feature.

Figure 7 shows a magnification of the wavelength range 4635-4655 $\AA$ of a combined primary plus secondary spectrum (weighted for sizes) and the effect of applying broadening mechanisms. The broadening is clearly dominated by rotation. Velocities were again calculated assuming a tidally locked and circular orbit with $2 \pi r_{\mathrm{s}} / P=58.6 \mathrm{~km} \mathrm{~s}^{-1}$ for the secondary. The synthetic spectra match the observation quite well, so no additional rotational broadening is required.
To explain the emission lines of C III at $\lambda 4649$ and 4651 to $4653 \AA$ the $\mathrm{C}$ abundance in the secondary needs to be set to $\epsilon_{\mathrm{C}}=+1.5$ dex solar in NLTE and even more in LTE. This seems unreasonable considering that the primary is $\mathrm{C}$ depleted during the CEE phase. It is also not compatible with observations outside the 4635-4655 $\AA$ broad emission feature, where synthetic spectra with $\mathrm{C}$ abundances that high predict unobserved emission lines.

The $\mathrm{N}$ abundance is not high enough to reproduce the size of the middle broad emission feature; this indicates that a higher $T_{\text {eff, }}$ is needed and that some $\mathrm{N}$ was accreted during the CEE, enriching the secondary's surface by approximately $+1 \mathrm{dex}$. This is reasonable, since, in contrast to $\mathrm{C}$, the primary is greatly enriched in $\mathrm{N}$ in its photosphere.

The abundance of $\mathrm{O}$ on the secondary is difficult to constrain, since the only emission line is flanked by stronger $\mathrm{C}$ lines (see Fig. 7). We use +1 dex for the models shown. From evolutionary considerations it need not be enriched.

The instrumental resolution is not high enough to resolve single lines from the broad emission feature originating from the secondary in Fig. 7. It might be feasible, however, to analyse the shape if one knows what lines are expected within the broad feature. In case of the double peak in the third broad emission feature, it seems that CIV fits the observation of the secondary's induced emission best for a $85000 \mathrm{~K}$ primary, while for the $75000 \mathrm{~K}$ and $95000 \mathrm{~K}$ model the shape is off-balance on the left. The last model hence suggests less heating or a larger separation. In contrast, there are N II and C II emission lines from the secondary day-side at the same wavelength as the $\mathrm{N} \mathrm{V}$ absorption $(\lambda 4603+4619 \AA)$ by the primary that add up to a worse overall fit. Increased heating results in more N III and C III, but the higher continuum changes the shape and hence does not reduce this problem. Combining these two considerations results in a $T_{\text {eff, p }}$ value of $80000-85000 \mathrm{~K}$, comparable to the light curve analysis of Pustynski \& Pustylnik (2005); Afşar \& Ibanoğlu (2008) and the revised value of BPH94.

Table 3 lists the model prediction for $T_{\mathrm{eq}, \mathrm{s}}$ on the day-side of the secondary depending on $T_{\text {eff, }}$ for a separation of $2.46 R_{\odot}$ and $T_{\text {eff, }}=3400 \mathrm{~K}$. Results vary on the order of $50-100 \mathrm{~K}$ for 1 dex abundance changes.

The typical temperature of the medium where the observed emission lines of heavy elements are formed is $21000-26000 \mathrm{~K}$. PB93 gives $25300 \pm 1000 \mathrm{~K}$ for the heated 


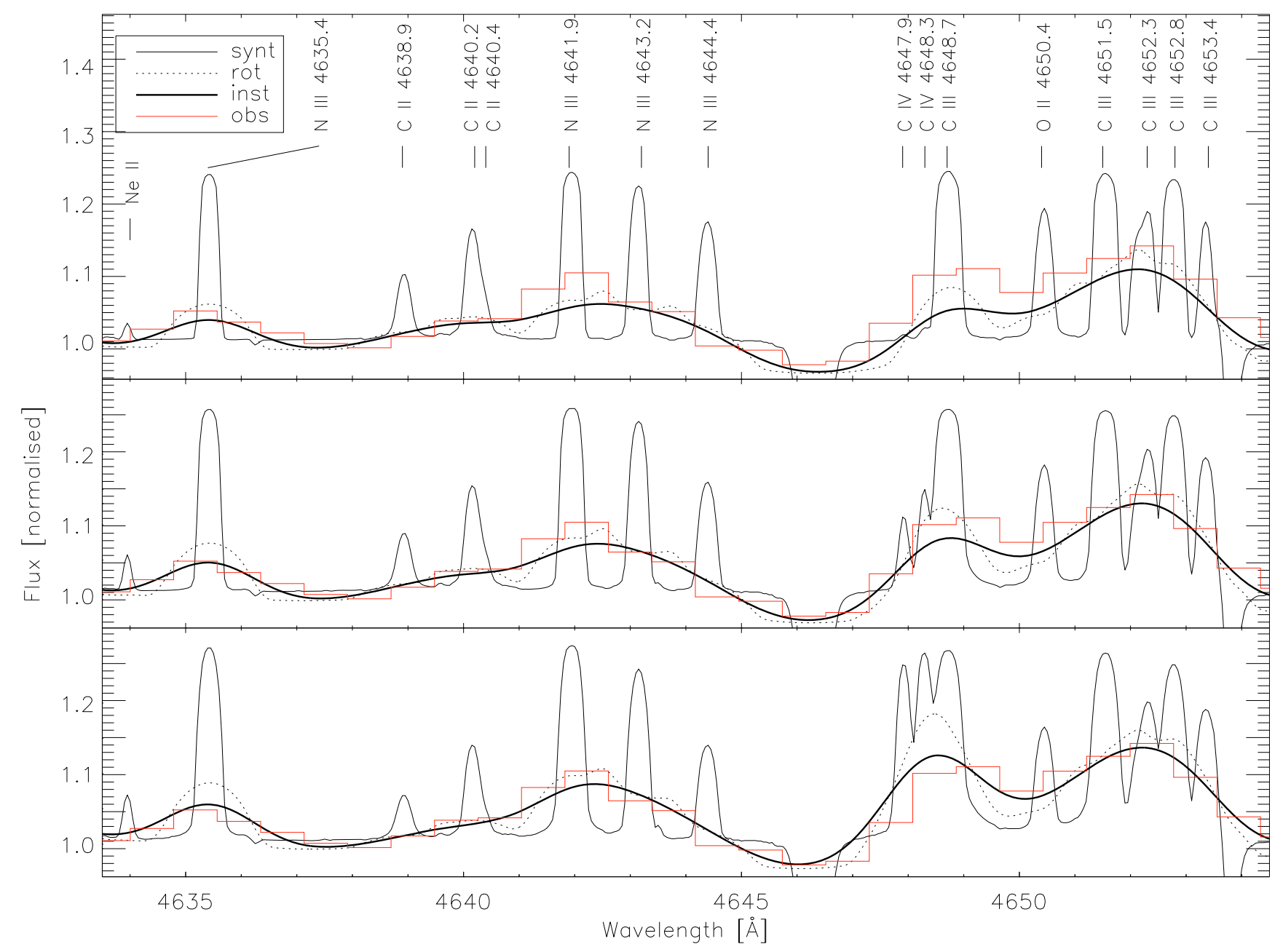

Fig. 7. Combined theoretical best-fit primary (for each $T_{\text {eff,p }}$ ) and $Z=+0.5$ dex plus CNO-increased secondary spectrum, weighted for the sizes of the components. The sharp emission features are from the pure synthetic spectrum (solid), to which first rotational (dotted) and then instrumental broadening (thick) is applied. The red/gray histogram is the observation. The primary's effective temperature used for irradiation is $75000 \mathrm{~K}$ (top), $85000 \mathrm{~K}$ (middle) and $95000 \mathrm{~K}$ (bottom), all other parameters of the secondary such as abundances are fixed, though these are increased by more than one order of magnitude relative to solar values in $\mathrm{C}$ and $\mathrm{N}$.

Table 3. $T_{\text {eff,p }}$ and model predicted $T_{\text {eq, s }}$.

\begin{tabular}{cc}
\hline \hline$T_{\text {eff,p }}$ & $T_{\text {eq, }}$ \\
\hline $75000 \mathrm{~K}$ & $23100 \mathrm{~K}$ \\
$85000 \mathrm{~K}$ & $26300 \mathrm{~K}$ \\
$95000 \mathrm{~K}$ & $29500 \mathrm{~K}$ \\
$105000 \mathrm{~K}$ & $32600 \mathrm{~K}$ \\
$115000 \mathrm{~K}$ & $35800 \mathrm{~K}$ \\
$125000 \mathrm{~K}$ & $39100 \mathrm{~K}$ \\
\hline
\end{tabular}

Using $R=2.46 R_{\odot}$ and $T_{\text {eff, }}=3400 \mathrm{~K}$.

day-side of the secondary of UU Sge, which would be consistent with a primary temperature of $78000-85000 \mathrm{~K}$ in our case and supports our own $T_{\text {eff, }}$ considerations above.

In this case the irradiation of the primary dominates the atmosphere of the secondary completely deeper than optical depth, $\tau=1$. The variation in $T_{\mathrm{eq}, \mathrm{s}}$ for changing $T_{\mathrm{eff}, \mathrm{s}}$, while $T_{\text {eff, } \mathrm{p}}=85000 \mathrm{~K}$ and $R=2.46 R_{\odot}$ are kept constant, is negligible. Comparing $T_{\text {eq, s }}$ for a model with $T_{\text {eff, }}=3400 \mathrm{~K}$ and one with $T_{\text {eff, s }}=6000 \mathrm{~K}$ results in a mere change of 20-30 K.

An interesting effect that was already observed by Brett \& Smith (1993) in irradiated models (10 $000 \mathrm{~K}$ blackbody primary) is that the optical depth at a given geometrical depth increases with increasing irradiative flux, i.e., the radiation makes the surface layers more opaque. We see a similar effect for primary temperatures up to roughly $85000 \mathrm{~K}$, at even higher temperatures the opposite occurs and the surface layers of the secondary start to become less opaque again, because some species become fully ionised.

The high difference between primary and secondary nightside flux contribution to the observed spectrum that allows one the decoupling of the primary from the secondary parameters in the first step of this analysis prevents a proper $T_{\text {eff, s }}$ estimate for the night-side of the secondary being made. At phase $\varphi \sim 0.0$ the spectrum is dominated by the primary and the night-side of the secondary cannot be fitted. The heat flux beyond the terminator in convective layers should adjust to a static solution if it is supposed to be the same star. An attempt to match the structure, i.e., the entropy per free particle, of the irradiated model day-side with hotter models of the undisturbed night-side was unsuccessful. The radiation field of the primary dominates the temperature structure of the day-side of the secondary deep into the photosphere, the convection zone is therefore pressed down into deeper layers and no common adiabat was found.

Figure 8 shows the structure of a secondary atmosphere, irradiated by the best-fit $T_{\text {eff, } \mathrm{p}}=85000 \mathrm{~K}$ primary. The temperature 


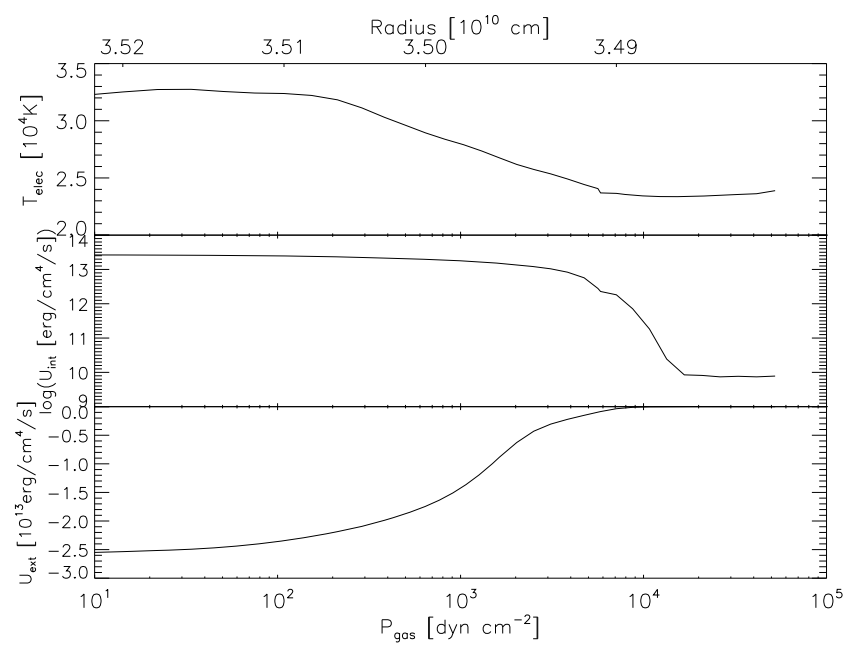

Fig. 8. The structure of the temperature and radiation field in the irradiated atmosphere at the substellar point. The small kink at $P_{\text {gas }}=$ $5 \times 10^{3} \mathrm{dyn}^{-2}$ is due to numerics. The upper panel shows electron temperature $T_{\text {elec }}$, the middle panel the logarithm of the internal radiation flux $u_{\mathrm{H}, \text { int }}$, and the lower panel the external radiation flux $u_{\mathrm{H} \text {,ext }}$. See text for details of the definition of the fluxes.

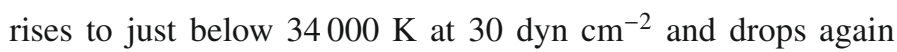
by about a third to deeper layers. Our model is calculated down to $5 \times 10^{4} \mathrm{dyn}_{\mathrm{cm}}^{-2}$. The lower panels of that figure characterise the radiation field: The middle panel shows the internal radiation flux $u_{\mathrm{H} \text {,int }}$. This is calculated as the radiation flux that would originate in an atmosphere with the temperature structure from the upper panel without any external radiation. At the inner boundary $u_{\mathrm{H} \text {, int }}$ is fixed, matching the blackbody radiation for the intrinsic, i.e., undisturbed, temperature of the secondary.

For each layer radiation is generated going in- and outwards. The radiation going inwards is reflected at the inner boundary condition and thus cancels out in the derivation of $u_{\mathrm{H}}$, int, whereas the radiation going outwards is summed up and therefore $u_{\mathrm{H} \text {, int }}$ increases monotonically outwards by more than three orders of magnitude. The energy lost exceeds the energy delivered from the inner boundary by far and the photosphere would cool down without an external energy source.

The lower panel in Fig. 8 shows the external radiation flux $u_{\mathrm{H} \text {, ext }}$. To obtain a measure of the influence of the external radiation field a full model including the internal flux and the irradiation is calculated. The difference between the radiation field obtained in this case and the internal radiation flux $\left(u_{\mathrm{H}, \text { int }}\right)$ is $u_{\mathrm{H} \text {, ext }}$. This characterises the layers where the incident radiation is reprocessed in the atmosphere. $u_{\mathrm{H} \text {, ext }}$ is negative, because it is directed inwards.

The plot does not contain reflected external irradiation, since this cancels out in the net flux $u_{\mathrm{H} \text {, ext }}$. In the thin outer layers of the atmosphere the optical depth is low, so only little flux is absorbed. Around a gas pressure of $10^{3} \mathrm{dyn} \mathrm{cm}^{-2}$, much of the external flux is absorbed, causing a temperature inversion, so deeper layers are cooler again. Between $10^{3} \mathrm{dyn}^{-2}$ and $2 \times 10^{3} \mathrm{dyn}^{-2}$ the external radiation flux deceases to $1 / e$ of the initial value, thus this can be taken as the depth to where the external radiation penetrates.

The ionisation in the outer layers is not collisionally dominated but the ionisation structure is given by the external radiation field, leading to an "over-ionisation". Figure 9 shows the dominant stages of ionisation for the ions of $\mathrm{C}, \mathrm{N}$, and $\mathrm{O}$. Although the temperature is only $25000 \mathrm{~K}$ at $10^{-2}$ dyn $\mathrm{cm}^{-2}$,

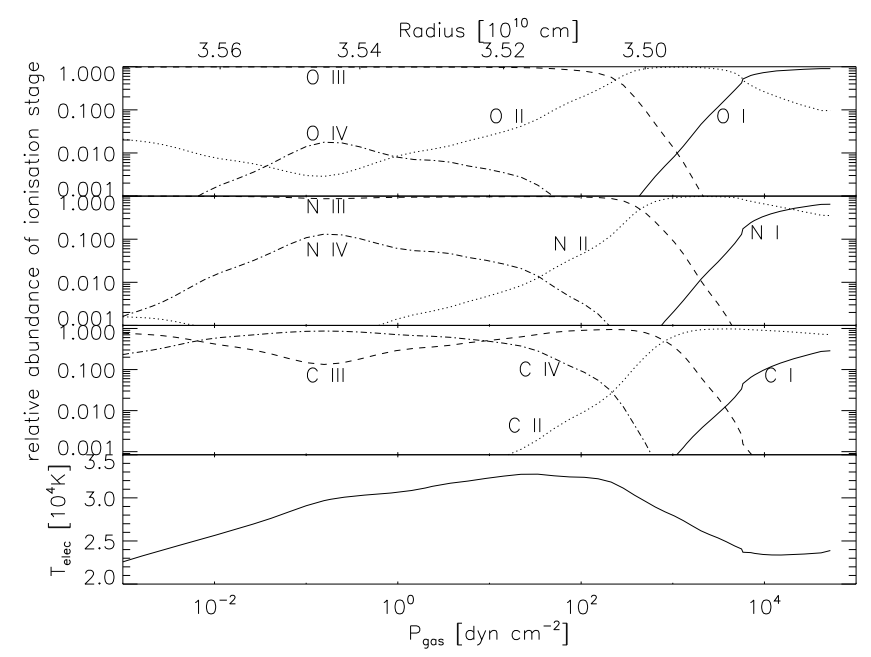

Fig. 9. The relative abundances of the most prominent ionisation stages for $\mathrm{C}, \mathrm{N}$ and $\mathrm{O}$. For comparison purposes the lower panel contains the temperature structure.

higher stages of ionisation ( $\mathrm{C} \mathrm{IV}, \mathrm{N}$ III and O III) prevail compared to the inner, denser layers at the same temperature, but the column mass of these elements is so small that they do not contribute any significant emission lines. Most of the spectral features above originate around $10^{3} \mathrm{dyn} \mathrm{cm}^{-2}$, where most of the incident energy is reprocessed. In this region $\mathrm{C}$ II and C III, N II, $\mathrm{N}$ III, and $\mathrm{O}$ II are most dominant. At deeper layers these ions recombine to neutral atoms, but the density and therefore the optical depth also increases, so that no line emission from atoms is observed. If the primary is either closer or hotter than expected than the stronger irradiation would lead to higher stages of ionisation in the crucial region around $10^{3} \mathrm{dyn}^{-2}$ and cause more emission lines which are not observed, e.g. O VI.

\section{Discussion}

In our model the secondary is tidally locked in a circular orbit, hence is rotating synchronously, because its calculated $v \sin i$ fits the observation and primary and secondary eclipses are equidistant in the light curve of PB93. The primary cannot be tidally locked, since additional broadening requires a speed-up by a factor of approximately $4-5$, which is still a reasonable rotational velocity for a subdwarf with radiative envelope that was spun up by its own contraction.

Remember that we have not included a wind in the model and that a turbulence on the surface can explain the additional broadening at least in parts.

We assume a circular orbit for simplicity, even though it is worth noting that within the framework of Zahn's theory, the synchronisation timescales are several orders of magnitude smaller than the circularisation timescales (Toledano et al. 2007). This ensures a uniform irradiation at a constant distance on the same side of the secondary, which itself is convective at least in layers the irradiation cannot penetrate. Also worth noting is that Zahn $(1977,1989)$ found the characteristic timescale for synchronisation as a function of $a$, the major semi-axis of the orbit, to be $\tau_{\text {sync }} \sim a^{6}$ for stars with convective envelopes and $\tau_{\text {sync }} \sim a^{8.5}$ for stars with with radiative envelopes, which might explain why the smaller, more compact and still contracting primary has not yet reacted to synchronisation.

An alternative mechanism has been proposed by Tassoul \& Tassoul (1992) where the spin down time for a given system 
depends only on the radius as $R^{-4.125}$. Again the larger secondary synchronises faster.

\subsection{Primary component}

Without any limb darkening or brightening effects included $T_{\text {eff, }}=75000 \mathrm{~K}$ is in between the results of PB93/BPH94 for the primary. It is in agreement with their error bars, given a fixed separation of $2.46 R_{\odot}$. We, however, favour a higher value of $80000-85000 \mathrm{~K}$, due to the effects on the broad emission feature on the secondary (see Fig. 7), even though this requires an enrichment of approximately $+1.5 \mathrm{dex}$ in $\mathrm{N}$ on the primary surface. This result resembles the light-curve solution by Pustynski \& Pustylnik (2005) and the new values by Afşar \& Ibanoğlu (2008).

In general, He rich sdO stars are also enriched in some metals, especially $\mathrm{C}$ and $\mathrm{N}$. This clearly indicates that the surface contains material burned in the $\mathrm{CNO}$ cycle as well as products of helium burning (Dreizler \& Murdin 2000). Within these nuclear burning processes $\mathrm{C}$ is turned into $\mathrm{N}$ by proton capture, probably benefited by some effect that mixes protons into deeper layers. Thus the abundance of $\mathrm{C}$ falls while the abundance of $\mathrm{N}$ increases $\left.\left({ }^{12} \mathrm{C}(\mathrm{p}, \gamma)\right)^{13} \mathrm{~N}\left(\mathrm{e}^{+}, v\right){ }^{13} \mathrm{C}(\mathrm{p}, \gamma){ }^{14} \mathrm{~N}\right)$. He is turned into $\mathrm{C}$ via the triple alpha $(3 \alpha)$ reaction, but $\mathrm{C}$ is destroyed in the outer parts of He-burning shells by the reaction ${ }^{12} \mathrm{C}(\alpha, \gamma){ }^{16} \mathrm{O}$. Hence $\mathrm{C}$ is depleted more than $\mathrm{N}$ is enriched. The sdO of UU Sge is decreased in $\mathrm{He}$ and especially enriched in $\mathrm{N}$ and depleted in $\mathrm{C}$ and $\mathrm{O}$. This can be understood as stripping the outer envelope layers down to a layer which was enriched in $\mathrm{N}$ during the CEE; the H-rich and He-rich layers, where the $3 \alpha$ process which produces $\mathrm{C}$ is most efficient, have been removed.

The production of ${ }^{14} \mathrm{~N}$ at the cost of $\mathrm{C}$ and $\mathrm{O}$ in the $\mathrm{CNO}$ cycle (de Greve \& Cugier 1989) is not unusual in the evolution of AGB stars during their third dredge up phase (see Herwig (2005) for a review). Enriched N and depleted C is also observed in WD+MS binaries as supernovae ( $\mathrm{SN}$ ) Ia progenitors (Langer et al. 2000). The depletion of He is most likely due to the CEE, i.e., the stripping of the outer layers.

Note that He is slightly underabundant in UU Sge for all our models. If a high $T_{\text {eff, }}$ is prescribed for the model then the He abundance is fitted closer to solar values.

$\mathrm{N} \mathrm{v} \lambda 4605$ is in emission for NLTE models with $T_{\text {eff, } \mathrm{p}} \geq$ $110000 \mathrm{~K}$. There are no known sdOs with such high temperatures (S. Dreizler, private communication). There is, however, PG1144+005 $\left(T_{\text {eff }}=150000 \mathrm{~K}\right.$ and $\left.\log g=6.5\right)$, which is a peculiar PG1159-like star that shows such N V emission lines at $\lambda 4603$ and $4619 \AA$ (Werner \& Heber 1991). Therefore this NLTE effect seems reasonable and suggests a lower $T_{\text {eff, }}$ than $100000 \mathrm{~K}$, probably even lower than 90 000-95000 K; therefore our model is contradicting the primary temperature of $120000 \mathrm{~K}$ found by Shimansky et al. (2008).

There is a problem with the surface gravity derived from He II $\lambda 4686 \AA$ which suggests a $\log g_{\mathrm{p}} \approx 4.5$, and we require a He depletion of -0.5 dex to fit all other He II absorption lines. Using the mass and radius from PB93 $\log g_{\mathrm{p}}$ of the primary is constrained to $5.2 \pm 0.2$ as mentioned before. Probably this is due to numerically instabilities in the treatment of the radiation pressure in the model, since it seems unlikely that the primary is much less massive or has a veil of reaccreted He within its Roche lobe that has not fallen back on the surface yet. At phase 0.5 this problem is solved by adding emissions from the day-side of the secondary and consistent with a $\log g_{\mathrm{p}}=5.0$ to 5.2. However, at phase 0.0 the night-side, even if set to $T_{\mathrm{eff}, \mathrm{s}}=6000 \mathrm{~K}$ (e.g. as would be due to a horizontal heat flux beyond the terminator), is too weak for the same correction. It is unclear whether the primary is really less compact than expected, or why otherwise this particular He II feature is weaker than expected, since there is no indication of any emission (e.g. by material within the secondary Roche lobe through which radiation is transmitted).

While main sequence $\mathrm{O}$ stars are known to exhibit a stellar wind, the atmospheres of most sdO stars can be regarded as hydrostatic. Of course, signatures of a stellar wind can be detected in the most luminous sdOs through P-Cygni profiles in UV resonance lines or through emission lines in the visible, but even in these stars all other lines originate from quasi-static layers of the atmosphere (Dreizler \& Murdin 2000).

According to radiation driven wind theory (Pauldrach et al. 1988) a star like UU Sge, which is close to the Eddington limit, should have a mass loss rate of $2 \times 10^{-8} M_{\odot} \mathrm{yr}^{-1}$ and a terminal velocity of $2000 \mathrm{~km} \mathrm{~s}^{-1}$. PB93 find, however, no P Cygni profiles in resonance lines of IUE data. This implies that the wind velocity is $<1000 \mathrm{~km} \mathrm{~s}^{-1}$. PB93 also inferred a stellar wind mass loss rate of $<1 \times 10^{-9} M_{\odot} \mathrm{yr}^{-1}$ by using the error in the period determination as an upper limit over nearly 12000 orbital circles.

We do not include limb darkening in our models, since PB93 find no limb darkening for primary temperatures in excess of $85000 \mathrm{~K}$, analysing the light curve around the ingress and egress from the primary minimum.

\subsection{Secondary component}

Webbink (1988) claimed, inferred from observations of the binary core of planetary nebulae, that the unevolved secondary star has been little disturbed by the CEE and resembles a normal main-sequence star. The night-side temperature of the secondary component, measured by BPH94, however, would normally indicate a spectral type of late $\mathrm{A}$ / early $\mathrm{F}$ and consequently a mass around $\sim 1.6 M_{\odot}$ and a radius around $\sim 1.5 R_{\odot}$, assuming it is on the main sequence. Hence the derived mass of $0.29 M_{\odot}$ and radius of $0.53 R_{\odot}$ imply that the evolutionary path of this object has been greatly influenced by the sdO star. This mass estimate indicates an M-type dwarf.

It is conceivable that this star has been stripped of its outer layers during the CEE and accreted some other material.

Due to the amount of material accreted and the short time since the CEE phase, the secondary may still be out of thermal equilibrium. The thermal relaxation time-scale of the disturbed outer layers of the secondary, once the common envelope is ejected, is $\sim 10^{4}$ years, comparable to the estimated age of the PN. This could explain why the secondary is oversized for its mass (PB93; Afşar \& Ibanoğlu 2008) and could mean that the internal structure of the secondary is inhomogeneous, i.e., the accreted material has not mixed in (Prialnik \& Livio 1985; Sarna $\&$ Ziolkowski 1988). Probably the heating on the day-side of the secondary also contributes to the inflation of the star.

Using the wind mass loss rate by PB93 no more than $10^{-5} M_{\odot}$ have been ejected by the primary during the PN lifetime. This is orders of magnitude below the required $\mathrm{N}$ mass needed to explain the abundances in the secondary, even for a pure $\mathrm{N}$ wind that is completely accreted.

Since the secondary is less massive than the primary (the mass ratio $q<1.0$ ) the system has been detached since the end of the CEE and will be, until gravitational wave radiation and magnetic stellar wind braking brings the secondary in contact with its Roche lobe again (Sarna et al. 1996). The secondary may resemble the composition of the primary. 
According to Marks \& Sarna (1998) the effect of accretion during CEE on the abundances is expected to be very small, the only significant difference being seen in the abundance of $\mathrm{N}$, which is increased by less than one order of magnitude. The secondary of UU Sge, however, displays an enrichment of not only $\mathrm{N}$ but also $\mathrm{C}$ and probably $\mathrm{O}$ in its photosphere compared to $Z_{\mathrm{s}}=+0.5$. It is oversized compared to a zero-age MS star of the same mass (BPH94). This might be understood as a layer of material accreted from the primary, possibly related to the last layer stripped there. Probably $\mathrm{C}$ and $\mathrm{N}$ were transferred to the secondary while $\mathrm{C}$ was still being transformed to $\mathrm{N}$ in the primary. Accreted material may still settle down to deeper layers in the secondary but not yet be mixed in, hence producing an unusual abundance in the currently visible spectrum.

In their calculations of the common envelope phase, Hjellming \& Taam (1991) found that the secondary accretes approximately $0.1 M_{\odot}$ of red giant envelope material before it expands to fill its Roche lobe. However this estimate is based on calculations by Taam \& Bodenheimer (1989) and highly dependent on the assumed efficiency of envelope ejection. Marks \& Sarna (1998) point out that once it has filled its Roche lobe, it loses most of the material accreted before the envelope is ejected such that the net gain in mass is approximately $0.01-0.06 M_{\odot}$. It is not clear which part of the envelope is predominantly accreted.

PB93 already noticed this similarity in spectra between UU Sge and V477 Lyr and absorption lines from the Balmer series, He II and N V ions while He I lines are absent. They point out that the strength of He II lines suggests that the Balmer lines are contaminated by other members of the He II series, i.e., $\mathrm{H} \beta+\mathrm{He}$ II $\lambda 4859, \mathrm{H} \gamma+\mathrm{He}$ II $\lambda 4338$ and $\mathrm{H} \delta+\mathrm{He}$ II $\lambda 4098 \AA$. However, our observations only contain $\mathrm{H} \gamma$.

The strong broad emission feature at $4635-4655 \AA$ is remarkably similar in shape to the V477 Lyr observation by Shimansky et al. (2008). It seems to be common to sdO+MS pre-CVs and is also the strongest emission between 3950 and $5100 \AA$. It is remarkably similar in shape to the V477 Lyr observation by Shimansky et al. (2008). This confirms the similarity of these two systems, as already pointed out by Ritter (1986) and PB93. There are, however, more smaller emission features visible which indicate that the secondary in V477 Lyr has greater influence on the total spectrum than the one in UU Sge. The broad feature is produced by very strong $\mathrm{C}$ and $\mathrm{N}$ emission lines in the secondary, as discussed in Sect. 5.2, while the rest of the spectrum is better fit by lower $\mathrm{C}$ and $\mathrm{N}$ abundances.

Emissions lines from a chromosphere are unlikely on the day-side due to the strong external radiation field. Also the contribution from a chromosphere on the night-side, if existent, is not likely to explain the missing emission for He II $\lambda 4686$ in the combined spectrum (Fuhrmeister, private communication).

\subsection{Evolution of UU Sge}

The evolution of UU Sge is still not completely understood. N is overabundant, indicating that the system reached the AGB, expelling the outer envelope and mixing $\mathrm{N}$ up to the observable surface. There was most likely no crucial interaction between the two stars until the primary envelope expanded to a size that it engulfed the secondary and started CEE.

This might be explained by an enrichment in $\mathrm{C}, \mathrm{N}$ and probably $\mathrm{O}$ that occurred during the CEE and only effects outer layers of the secondary, which might not yet be in thermal equilibrium. Also the secondary might have accreted $\mathrm{C}$ from an outer layer ( $3 \alpha$ process) while the $\mathrm{CNO}$ cycle in the primary was still
Table 4. Final results (see discussion):

\begin{tabular}{ccc}
\hline \hline & Primary & Secondary \\
\hline$T_{\text {eff }}$ & 80000 to $85000 \mathrm{~K}$ & $(6000$ to $7000 \mathrm{~K})$ \\
$T_{\text {eq }}$ & n.a. & 24000 to $26000 \mathrm{~K}$ \\
$\log g$ & $(4.5), 5.0$ to 5.2 & $(4.45)$ \\
$\epsilon_{\mathrm{He}}$ & -0.2 to $-0.4 \mathrm{dex}$ & n.a. \\
$\epsilon_{\mathrm{C}}$ & $-0.2 \mathrm{dex}$ & +1.0 to $+1.5 \mathrm{dex}$ \\
$\epsilon_{\mathrm{N}}$ & $+1.2 \mathrm{dex}$ and more & $\approx+1.0 \mathrm{dex}$ \\
$L=4 \pi \sigma r^{2} T_{\mathrm{eff}}^{4}$ & 1 to $2 \times 10^{37} \mathrm{erg} \mathrm{s}^{-1}$ & 1 to $3 \times 10^{33} \mathrm{erg} \mathrm{s}^{-1}$ \\
$L=4 \pi \sigma r^{2} T_{\text {eq }}^{4}$ & n.a. & 2 to $5 \times 10^{35} \mathrm{erg} \mathrm{s}^{-1}$ \\
\hline
\end{tabular}

working to convert $\mathrm{C}$ into $\mathrm{N}$, explaining why this element is not found enriched on the primary, too.

Another possibility, although unlikely, is that the hot, oversized companion indicates that there were two AGB phases in the system and that the first was suppressed due to a too high mass-loss rate of the secondary. This would be another explanation for enrichment of heavier elements than $\mathrm{He}$ on the secondary, especially why there is $\mathrm{C}$ present. However, the secondary is too cold and its mass is too low to support this idea, since there is no proper mechanism known and its spectra look too ordinary. The primary could have gone through an earlier CEE of course, too, losing its envelope in more steps than just one.

The primary was stripped of its $\mathrm{H}$-rich and He-rich layers during the CEE, exposing a shell of $\mathrm{N}$ underneath. Both components display lines with peculiar effects: the He II $\lambda 4686$ absorption suggests a layer of $\mathrm{He}$ on top of the $\mathrm{N}$ on the primary, and a slightly lower $\log g_{\mathrm{p}}$ or some unexplained emission from the secondary; the broad emission feature on the secondary that reacts uniquely to abundance changes and is the strongest emission feature within the entire wavelength range of the observation.

The broad emission feature is visible also in the other irradiated systems, e.g. the secondary of V477 Lyr (Shimansky et al. 2008), which is also 2.0-2.5 times larger than the radius of a zero-age MS star with comparable mass (BPH94). This suggests a non-unique mechanism due to a similar evolution in both systems.

Table 4 displays the derived parameters of UU Sge from Sect. 5 .

\section{Summary}

We modelled both components of UU Sge with our state-of-theart stellar atmosphere code PHOENIX, which treats the radiative transfer in an atmosphere self-consistently in the presence of an external radiation field and uses the newest extensive set of opacities currently available. In this respect our work goes beyond previous work.

Our analysis provides the temperature range of the primary and investigates the heating of the secondary's day-side. We find evidence for a large $\mathrm{N}$ enrichment on the primary, a depletion of $\mathrm{C}$ and $\mathrm{O}$, and an upper limit for Si that is less than +0.3 dex solar. The lower $\mathrm{He}$ abundance in the $\mathrm{sdO}$ originates most likely from the loss of its envelope.

The observed broad emission blend at $4635-4655 \AA$ in the secondary is stronger than the theoretical result by a factor of 3-5, which is indicative of the strong effects of "overionisation" by external radiation in high atmospheric layers for the $\mathrm{CNO}$ elements. This might be due to pollution of material accreted from the primary, which has not yet settled down 
to lower layers in the oversized secondary and explain $\mathrm{C}$ and $\mathrm{N}$ enrichment.

It is obvious that both stars have been greatly influenced in their evolution by their companion. The highly enriched metals observed in the primary and secondary could indicate that there is no mixing in the outer layers, since any counter mechanism that removes $\mathrm{N}$ into deeper layers would greatly decrease its abundance and stars would not produce such peculiar line strengths.

Convection on the day-side of the secondary is suppressed by the irradiation, the heat flux is dominated by radiation throughout the entire photosphere. Observationally the night-side appears to be heated by a horizontal flux, resulting in an earlier spectrum then expected for the secondary mass. Without a horizontal component of convective motion or radiative transfer (a 3D model), we cannot model the transfer of heat from layers of the irradiated half-sphere towards the undisturbed night-side, thus we expect that motion beyond the limits of our model is responsible for the heating of the secondary night-side.

Further analysis of UV and IR spectra could improve the disentangling of primary and secondary spectra, since the UV is dominated by the sdO, while the companion main source lies in IR. An interesting line in PG1144+005 is N V $\lambda 4925 \AA$ (high $l$, $6 \rightarrow 7$ transition), which is a strong emission line and could be of considerable strength in UU Sge, too, hence allowing us to confirm the $\mathrm{N}$ enrichment independently to the doublet used here.

Another fit to a secondary night-side only spectrum during primary eclipse could help to test the M-dwarf thesis, to find the horizontal heat flux beyond the terminator and to check for $\mathrm{C}$, $\mathrm{N}$ and probably $\mathrm{O}$ enhancement on the surface of the secondary without the effect of irradiation.

Acknowledgements. We thank Stefan Czesla, Stefan Dreizler, Birgit Fuhrmeister, Roy Østensen, Jan Robrade, Brian Skiff and the unknown referee for helpful comments. A.C.W. was supported by the DFG (Deutsche Forschungsgemeinschaft), project number HA 3457/7-1. Lowell Observatory provided hospitality, volleyball games and scientific support to ACW while this paper was written. Calculations were performed at the Hamburger Sternwarte Delta Opteron Cluster ("Nathan") financially supported by the DFG and the State of Hamburg; at the Lowell Observatory's Apple Xeon Cluster ("Cuba"); and at the Höchstleistungs Rechenzentrum Nord (HLRN-II) Altix Computesystem ("ICE" and "XE"). We would like to thank these institutions for a generous allocation of computer time.

\section{References}

Abell, G. O. 1966, ApJ, 144, 259

Afşar, M., \& Ibanoğlu, C. 2008, MNRAS, 391, 802

Allard, F., Hauschildt, P. H., Alexander, D. R., Tamanai, A., \& Schweitzer, A. 2001, ApJ, 556, 357

Asplund, M., Grevesse, N., \& Sauval, A. J. 2005, in Cosmic Abundances as Records of Stellar Evolution and Nucleosynthesis, ASP Conf. Ser., 336, 25 Aufdenberg, J. P. 2001, PASP, 113, 119

Barman, T. S., Hauschildt, P. H., \& Allard, F. 2004, ApJ, 614, 338

Barman, T. S., Hauschildt, P. H., \& Allard, F. 2005, ApJ, 632, 1132

Beer, M. E., \& Podsiadlowski, P. 2002, MNRAS, 335, 358

Beer, M. E., Dray, L. M., King, A. R., \& Wynn, G. A. 2007, MNRAS, 375, 1000

Bell, S. A., Pollacco, D. L., \& Hilditch, R. W. 1994, MNRAS, 270, 449
Bond, H. E. 1976, PASP, 88, 192

Bond, H. E., Liller, W., \& Mannery, E. J. 1978, ApJ, 223, 252

Brett, J. M., \& Smith, R. C. 1993, MNRAS, 264, 641

Budding, E. 1981, in Photometric and Spectroscopic Binary Systems, ed. E. B. Carling, \& Z. Kopal, 405

Budding, E., \& Kopal, Z. 1980, Ap\&SS, 73, 83

Claret, A., \& Giménez, A. 2001, in Lecture Notes in Physics, Binary Stars: Selected Topics on Observations and Physical Processes, ed. F. C. Lázaro, \& M. J. Arévalo (Berlin: Springer Verlag), 563, 1

de Greve, J. P., \& Cugier, H. 1989, A\&A, 211, 356

de Kool, M., \& Ritter, H. 1993, A\&A, 267, 397

Dreizler, S., \& Murdin, P. 2000, Encyclopedia of Astronomy and Astrophysics Eggleton, P. P. 1983, ApJ, 268, 368

Exter, K. M., Pollacco, D. L., Maxted, P. F. L., Napiwotzki, R., \& Bell, S. A. 2005, MNRAS, 359, 315

Hauschildt, P. H., \& Baron, E. 1999, J. Comput. Appl. Math., 109, 41

Herwig, F. 2005, ARA\&A, 43, 435

Hjellming, M. S., \& Taam, R. E. 1991, ApJ, 370, 709

Hoffleit, D. 1932, Harvard College Observatory Bulletin, 887, 9

Hubeny, I., \& Lanz, T. 2000, http://nova.astro.umd.edu/Synspec43/ synspec-stark.html

Iben, I., \& Tutukov, A. V. 1989, in Planetary Nebulae, ed. S. Torres-Peimbert, IAU Symp., 131, 505

Iben, I. J., \& Livio, M. 1993, PASP, 105, 1373

Iben, I. J., \& Tutukov, A. V. 1993, ApJ, 418, 343

Langer, N., Deutschmann, A., Wellstein, S., \& Höflich, P. 2000, A\&A, 362, 1046

Livio, M. 1996, in The Origins, Evolution, and Destinies of Binary Stars in Clusters, ed. E. F. Milone \& J.-C. Mermilliod, ASP Conf. Ser., 90, 291

Marks, P. B., \& Sarna, M. J. 1998, MNRAS, 301, 699

Marsh, T. R. 2000, New Astron. Rev., 44, 119

Miller, J. S., Krzeminski, W., \& Priedhorsky, W. 1976, IAU Circ., 2974, 2

Moehler, S., Landsman, W., \& Napiwotzki, R. 1998, A\&A, 335, 510

Nelemans, G., \& Tout, C. A. 2005, MNRAS, 356, 753

Paczynski, B. 1976, in Structure and Evolution of Close Binary Systems, ed. P. Eggleton, S. Mitton, \& J. Whelan, IAU Symp., 73, 75

Pauldrach, A., Puls, J., Kudritzki, R. P., Mendez, R. H., \& Heap, S. R. 1988, A\&A, 207, 123

Pollacco, D. L., \& Bell, S. A. 1993, MNRAS, 262, 377

Prialnik, D., \& Livio, M. 1985, MNRAS, 216, 37

Pringle, J. E., \& Wade, R. A. (eds) 1985, Interacting binary stars (Cambridge: Cambridge University Press)

Pustynski, V.-V., \& Pustylnik, I. 2005, in 14th European Workshop on White Dwarfs, ed. D. Koester, \& S. Moehler, ASP Conf. Ser., 334, 639

Rasio, F. A., \& Livio, M. 1996, ApJ, 471, 366

Ritter, H. 1986, A\&A, 169, 139

Sandquist, E. L., Taam, R. E., Chen, X., Bodenheimer, P., \& Burkert, A. 1998, ApJ, 500, 909

Sarna, M. J., \& Ziolkowski, J. 1988, Acta Astron., 38, 89

Sarna, M. J., Marks, P. B., \& Connon Smith, R. 1996, MNRAS, 279, 88

Shimansky, V. V., Pozdnyakova, S. A., Borisov, N. V., et al. 2008, Astron. Lett., 34,423

Taam, R. E., \& Bodenheimer, P. 1989, ApJ, 337, 849

Taam, R. E., \& Ricker, P. M. 2006, ArXiv Astrophysics e-prints

Taam, R. E., \& Sandquist, E. L. 2000, ARA\&A, 38, 113

Tassoul, J.-L., \& Tassoul, M. 1992, ApJ, 395, 259

Toledano, O., Moreno, E., Koenigsberger, G., Detmers, R., \& Langer, N. 2007, A\&A, 461, 1057

van Winckel, H. 2003, ARA\&A, 41, 391

Walton, N. A., Walsh, J. R., \& Pottasch, S. R. 1993, A\&A, 275, 256

Warner, B. 1995, Cataclysmic variable stars (Cambridge, New York: Cambridge University Press), Cambridge Astrophys. Ser.

Webbink, R. F. 1988, in Colloquium on Critical Observations Versus Physical

Models for Close Binary Systems, 403

Webbink, R. F. 2007, ArXiv e-prints, 704

Werner, K., \& Heber, U. 1991, A\&A, 247, 476

Zahn, J.-P. 1977, A\&A, 57, 383

Zahn, J.-P. 1989, A\&A, 220, 112 